\title{
TU/e EmonOWEN

\section{Entrapped air bubbles in piezo-driven inkjet printing: their effect on the droplet velocity}

Citation for published version (APA):

Jong, de, J., Jeurissen, R. J. M., Borel, H., Berg, van den, M., Versluis, M., Wijshoff, H. M. A., Prosperetti, A., Reinten, H., \& Lohse, D. (2006). Entrapped air bubbles in piezo-driven inkjet printing: their effect on the droplet velocity. Physics of Fluids, 18(12), 121511-1/7. https://doi.org/10.1063/1.2397015

DOI:

10.1063/1.2397015

Document status and date:

Published: 01/01/2006

\section{Document Version:}

Publisher's PDF, also known as Version of Record (includes final page, issue and volume numbers)

\section{Please check the document version of this publication:}

- A submitted manuscript is the version of the article upon submission and before peer-review. There can be important differences between the submitted version and the official published version of record. People interested in the research are advised to contact the author for the final version of the publication, or visit the $\mathrm{DOI}$ to the publisher's website.

- The final author version and the galley proof are versions of the publication after peer review.

- The final published version features the final layout of the paper including the volume, issue and page numbers.

Link to publication

\section{General rights}

Copyright and moral rights for the publications made accessible in the public portal are retained by the authors and/or other copyright owners and it is a condition of accessing publications that users recognise and abide by the legal requirements associated with these rights.

- Users may download and print one copy of any publication from the public portal for the purpose of private study or research.

- You may not further distribute the material or use it for any profit-making activity or commercial gain

- You may freely distribute the URL identifying the publication in the public portal.

If the publication is distributed under the terms of Article 25fa of the Dutch Copyright Act, indicated by the "Taverne" license above, please follow below link for the End User Agreement:

www.tue.nl/taverne

Take down policy

If you believe that this document breaches copyright please contact us at:

openaccess@tue.nl

providing details and we will investigate your claim. 


\title{
Entrapped air bubbles in piezo-driven inkjet printing: Their effect on the droplet velocity
}

\author{
Jos de Jong, Roger Jeurissen, and Huub Borel \\ Physics of Fluids Group, Faculty of Science and Technology and Burgers Center of Fluid Dynamics, \\ University of Twente, P.O. Box 217, 7500 AE Enschede, The Netherlands
}

Marc van den Berg, Herman Wijshoff, and Hans Reinten

Océ Technologies B.V., P.O. Box 101, 5900 MA Venlo, The Netherlands

Michel Versluis, Andrea Prosperetti, ${ }^{a)}$ and Detlef Lohse

Physics of Fluids Group, Faculty of Science and Technology, Burgers Center of Fluid Dynamics, MESA + Institute for Nanotechnology and Institute of Mechanics, Processes and Control-Twente (IMPACT), University of Twente, P.O. Box 217, 7500 AE Enschede, The Netherlands

(Received 28 April 2006; accepted 25 August 2006; published online 8 December 2006)

\begin{abstract}
Air bubbles entrapped in the ink channel are a major problem in piezo-driven inkjet printing. They grow by rectified diffusion and eventually counteract the pressure buildup at the nozzle, leading to a breakdown of the jetting process. Experimental results on the droplet velocity $u_{\text {drop }}$ as a function of the equilibrium radius $R_{0}$ of the entrained bubble are presented. Surprisingly, $u_{\text {drop }}\left(R_{0}\right)$ shows a pronounced maximum around $R_{0}=17 \mu \mathrm{m}$ before it sharply drops to zero around $R_{0}=19 \mu \mathrm{m}$. A simple one-dimensional model is introduced to describe this counterintuitive behavior which turns out to be a resonance effect of the entrained bubble. (c) 2006 American Institute of Physics.
\end{abstract}

[DOI: $10.1063 / 1.2397015]$

\section{INTRODUCTION}

The number of possible applications of drop-on-demand (DOD) printing has increased considerably in the last few years. Beyond printing of text and pictures, the technique will be or has already been applied in diagnostics, the pharmaceutical industry, and the manufacturing of solar cells and small and cheap devices. ${ }^{1,2}$ Stability of the inkjet printing process and its reproducibility is crucial for most of these applications. Moreover, a large jetting frequency is desirable, in order to reduce the printing time.

Under normal conditions drop-on-demand piezo-electric inkjet printing $^{3-9}$ fulfills all the requirements with respect to stability and reproducibility. However, under certain conditions an air bubble can be entrained in the nozzle, ${ }^{10-12}$ in particular at large jetting frequencies beyond $20 \mathrm{kHz}$. This air bubble grows by rectified diffusion: ${ }^{13-16}$ While at pressure maxima air is squeezed out of the bubble, this loss is overcompensated at the pressure minima when the bubble expands, resulting in a net gas diffusion into the bubble. The bubble growth first leads to a modification of the drop production process and ultimately to the breakdown of the jetting. In Ref. 12 we have introduced a method to acoustically monitor the inkjet channel, using the piezo as a sensor. In this way we could identify two different scenarios how bubbles are entrained at the nozzle and what their long-time effect is on the jetting. However, a direct optical observation of the entrained bubbles was not possible, as standard inkjet channels are not optically accessible.

In this paper we overcome this restriction by introducing

\footnotetext{
a) Also at Department of Mechanical Engineering, Johns Hopkins University, Baltimore, Maryland 21218.
}

a glass channel and a glass nozzle plate, so that direct observation of the entrained bubble and its radial and translational dynamics becomes possible. The aim of the paper is to correlate the bubble size (characterized by its equilibrium radius $R_{0}$ at standard static pressure) with the droplet velocity $u_{\text {drop. }}$. We will find that surprisingly the droplet velocity first increases with increasing bubble size, namely from about $1.5 \mathrm{~m} / \mathrm{s}$ without any air entrainment to about $2.5 \mathrm{~m} / \mathrm{s}$ for an air bubble with $R_{0}=17 \mu \mathrm{m}$. Then it decreases sharply and at a bubble radius of $R_{0}=19 \mu \mathrm{m}$ the jetting breaks down.

The paper is organized as follows: In Sec. II we briefly introduce the experimental setup. The main section is Sec. III, where we present the correlation results between $u_{\text {drop }}$ and the bubble size $R_{0}$. In Sec. IV we introduce a simple one-dimensional model to qualitatively account for the observations. The comparison with the experimental data is favorable (Sec. V). Section VI contains the conclusions and an outlook towards future work.

\section{EXPERIMENTAL SETUP}

The printheads under consideration in this paper are side-shooter printheads developed by Océ and used for professional printing. The schematic setup of the printhead is depicted in Fig. 1. ${ }^{12}$ The channel block is a graphite block with ink channels inside. The ink reservoir is connected to the channel and can be set at a specified pressure. In the rectangular ink channel one of the four walls is formed by a piezo element. Under the action of an applied voltage, the piezo first contracts increasing the volume of the ink channel thereby reducing the pressure and drawing in ink from the reservoir. During the second part of the pulse, the piezo expands, reducing the volume of the channel and ejecting a 


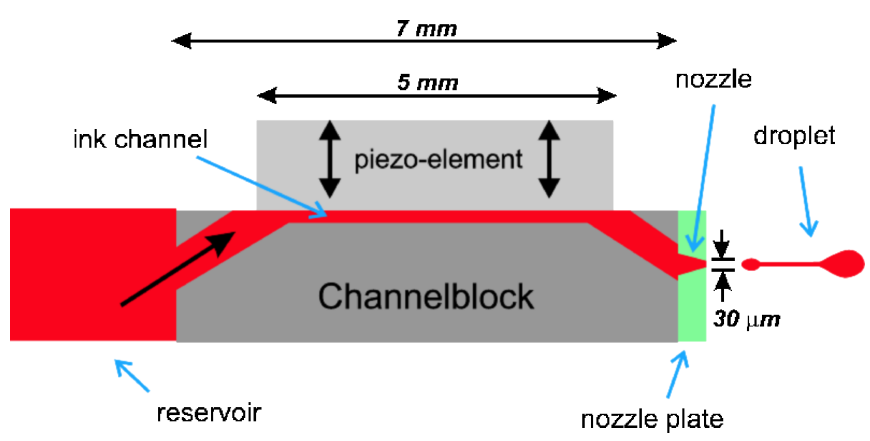

FIG. 1. (Color online) Sketch of the geometry of one ink channel of the printhead, which typically contains 256 of such channels. Only one channel on the printhead is actuated in our experiments. The pressure controlled reservoir supplies ink to the rectangular ink channel with a length of $7 \mathrm{~mm}$, width of $200 \mu \mathrm{m}$, and height of $150 \mu \mathrm{m}$. The piezoelement of length of $5 \mathrm{~mm}$ is covered with a foil of $20 \mu \mathrm{m}$ thickness, which is in direct contact to the ink. The nickel nozzle plate has round openings of diameter $30 \mu \mathrm{m}$, which serve as nozzles. The ejected droplets have a diameter of typically $35 \mu \mathrm{m}$.

drop. The pressure waves generated by the piezo travel towards the ink reservoir, where they are reflected out of phase, and to the nozzle, where they are reflected in phase. The ink is pressed out through a $30 \mu \mathrm{m}$ diameter electroformed nickel nozzle.

The piezo is actuated with $6 \mu$ s trapezoidal pulses with a repetition rate of $10 \mathrm{kHz}$, corresponding to one pulse every $100 \mu \mathrm{s}$, implying a $10 \mathrm{kHz}$ firing frequency of the droplets. More details of the driving protocol are given in Ref. 12. The transfer function of the piezo voltage, $V_{\text {act }}$, to the (maximum) nozzle velocity $u_{n}$ can be calculated from viscous acoustics in pipes, as described, e.g., by Tijdeman. ${ }^{17}$ As shown in Fig. 2 , in our printhead the dominant frequency is $f=60 \mathrm{kHz}$.

To optically monitor the entrapped air bubbles, the standard printhead just described was modified. The nickel nozzle plate was replaced with (i) a glass connection channel and, in addition, (ii) a glass nozzle plate glued to it; see Fig. 3(a) for a sketch, Fig. 3(b) for a photograph, and Fig. 3(c) for

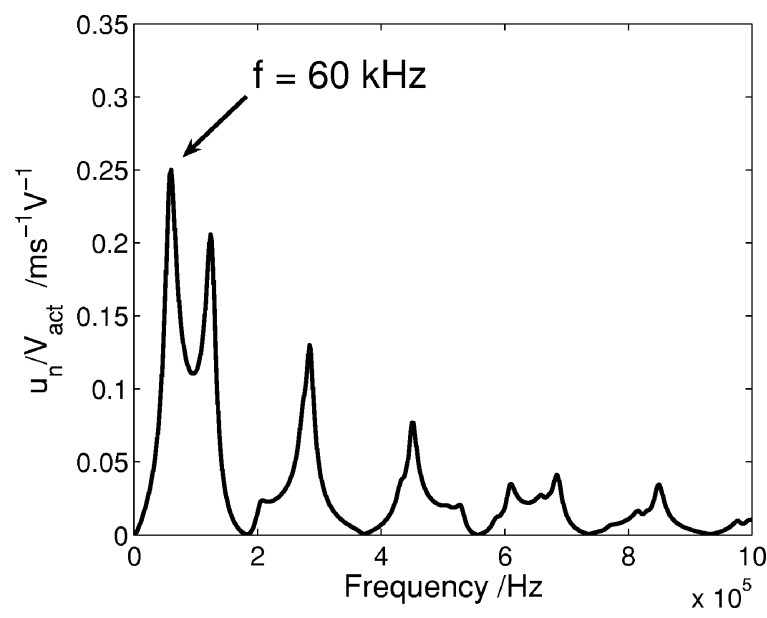

FIG. 2. The transfer function for the printhead under consideration showing the dominant frequency at $f=60 \mathrm{kHz} . u_{n}$ is the nozzle velocity and $V_{\text {act }}$ is the applied actuation voltage. The transfer function is calculated according to Tijdeman's model (Ref. 17) which from various measurements in other channels is known to well represent the experimental one. an enlargement of the entrained bubble. (i) The glass connection channel built by Micronit Microfluidics B.V. (Ref. 19) is constructed from a glass plate with a thickness of $400 \mu \mathrm{m}$. The channel was obtained by powder-blasting the glass plate from both sides, leading to an hourglass shape with a waist diameter of approximately $80 \mu \mathrm{m}$ and an inlet/outlet diameter of $250 \mu \mathrm{m}$. (ii) The glass nozzle plate was cut from a $70 \mu \mathrm{m}$ thick glass plate, which again was powder-blasted from both sides. The result is a conical nozzle shape with a diameter of about $50 \mu \mathrm{m}$ at the channel exit and $30 \mu \mathrm{m}$ at the nozzle end.

The nozzle plate and the connection channel are glued together to prevent leakage. The plates are then positioned onto a standard printhead, replacing the nickel nozzle plate. The glass is kept in place by small magnets, which push it against the printhead. To permit visualization of the air bubbles, a transparent ink was used. A continuous light source illuminates the glass from the back. A Phantom V7 high speed camera records the ejected droplets and the entrained air bubble at the same time. Due to the limited focal depth of the optical system, small bubbles in the ink channel can be visualized without optical distortions. For the chosen actuation voltage of $180 \mathrm{~V}$, the droplet velocity without entrapped bubbles is $u_{\text {drop }}=1.05 \mathrm{~m} / \mathrm{s}$.

In order to start the experiment we cause the entrapment of an air bubble as described in Ref. 12. Under the action of the oscillating pressure in the channel, the bubble grows by rectified diffusion ${ }^{13-15}$ and causes the droplet formation to stop. At this point the acoustic field is switched off and the bubble starts dissolving. Shortly before the dissolution is complete, actuation is resumed and the high speed camera is triggered, recording the growth of the bubble and its effect on the ejection of drops.

In Fig. 4 we show an example of the time evolution of a bubble obtained in this way. We first let a bubble of initial size $R_{0}=26 \mu \mathrm{m}$ dissolve; there is no actuation. The dissolution rate is constant at about $0.5 \mathrm{pl} / \mathrm{s}$, as seen from Fig. 4(b). At $t=165 \mathrm{~s}$ the actuation is switched on, leading to immediate jetting through the nozzle. The bubble which had nearly completely dissolved then starts to grow by rectified diffusion. Note the fast growth of the bubble compared to its dissolution. Once it has reached an equilibrium radius of about $19 \mu \mathrm{m}$, jetting breaks down. This experiment shows the strong influence of the size of the entrained bubble on the jetting. Note that if we had waited some tens of seconds longer before switching on the actuation, the bubble nucleus would have completely vanished, and the nozzle would have resumed jetting without any problem, until the occurrence of the next bubble entrainment. ${ }^{12}$

The recorded images of the ejected droplets and oscillating bubbles are analyzed with a gray-level threshold to determine the location of the edges of the droplets and bubbles. The images of both drops and bubbles only consist of some tens of pixels, limiting the accuracy of the size determination for smaller bubbles. Other sources of errors are optical diffraction and the assumed sphericity of the droplets and bubbles in the digital image analysis. For the ejected drops and for the bubbles within the channel (away from the walls) we do not have any indication of deviations from sphericity. 


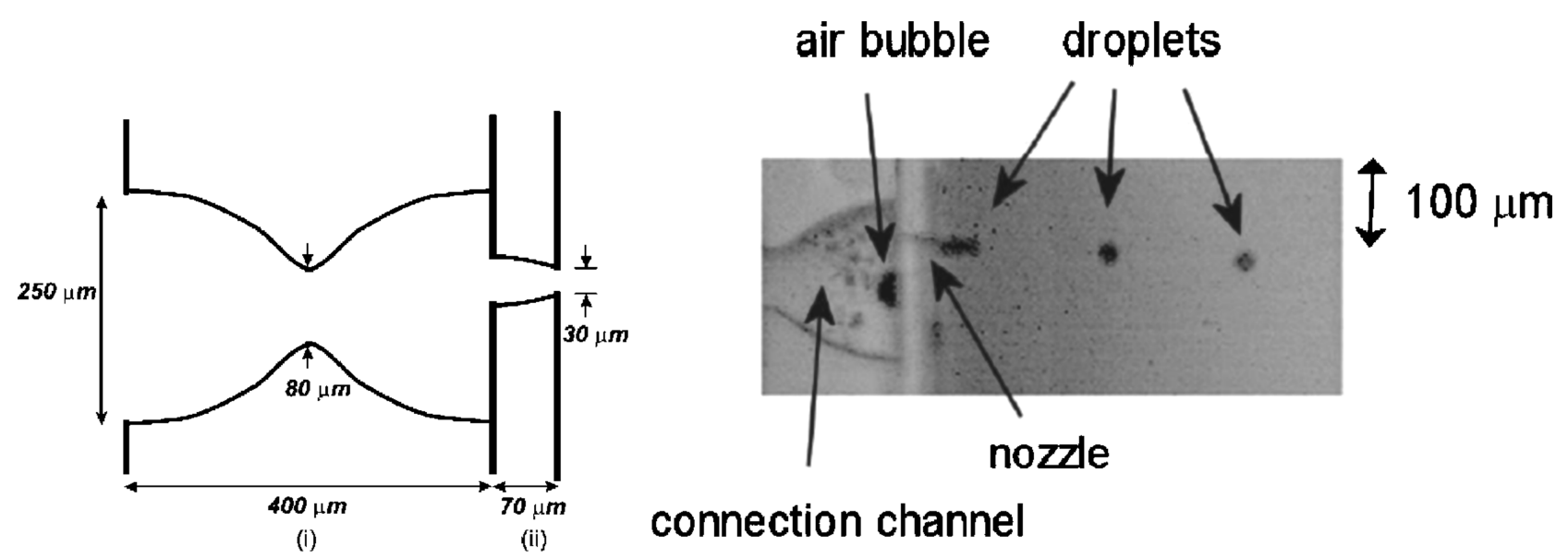

(a)

(b)

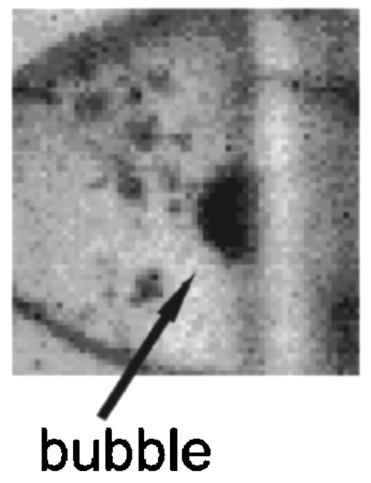

(c)

FIG. 3. (a) Sketch of (i) the glass connection channel with (ii) the glass nozzle plate glued to it. (b) Photograph of half of the glass connection channel and the glass nozzle plate under operation. An air bubble is present in the connection channel close to the nozzle and droplets are ejected. The multimedia part of this figure shows a high-speed movie (taken at 40000 frames per second) of the oscillating entrained bubble and the ejected droplets. (c) An enlargement of the bubble in (b). Here the arrow points at the bubble, that is pressed against the glass nozzle plate. The little dark dots left to the bubble originate from dirt on the glass connection channel (enhanced online).

However, the bubbles pushed against the glass nozzle plate seem to be slightly nonspherical.

During the experiments the jetting frequency is kept constant at $10 \mathrm{kHz}$. The frame rate of the camera is four times as high, $40 \mathrm{kfps}$. Therefore, 4 frames are acquired during one acoustic cycle, each at a different phase. To prevent motion blur the exposure time was set to $3 \mu \mathrm{s}$.

\section{EXPERIMENTAL RESULTS: BUBBLE SIZE AND JET VELOCITY}

In Fig. 5(a) the droplet velocity is displayed as a function of time. The actuation is started at $t=0 \mathrm{~s}$. Up to $t=0.2 \mathrm{~s}$, the droplet velocity increases, reaching a maximum of $2.5 \mathrm{~m} / \mathrm{s}$. Then the droplet velocity gradually decreases to $u_{\text {drop }}=1 \mathrm{~m} / \mathrm{s}$ at $t=0.9 \mathrm{~s}$. A small amplitude oscillation of $f=50 \mathrm{~Hz}$ is superimposed onto the droplet velocity, reflecting the ac frequency of the devices. This effect is negligible compared to the effect of the entrained air bubble.

When the radius of the air bubble is plotted in Fig. 5(b), it is evident that the air bubble grows over time by rectified diffusion. The scatter in the bubble radius is found to be quite large, due to the low contrast in the images and because multiple bubble radii are measured over one acoustic cycle. When we compensate for the latter by plotting the average radius (over 40 bubble radii) the bubble growth becomes more obvious as depicted in Fig. 5(c). Combining Figs. 5(a) and 5(c) results in the droplet velocity $u_{\text {drop }}$ as a function of the bubble radius $R_{0}$, Fig. $5(\mathrm{~d})$. This figure is the main experimental result of this work. Surprisingly, $u_{\text {drop }}$ first increases with increasing bubble radius. Around $R_{0} \approx 17 \mu \mathrm{m}$ the droplet velocity has a pronounced maximum before dropping sharply and jetting breaks down for bubble radii around $19 \mu \mathrm{m}$.

To estimate the error in the bubble radius arising from averaging over only four frames per cycle, we numerically model the bubble oscillations with the Rayleigh-Plesset equation ${ }^{13}$ and then apply the very same averaging procedure as in the experiment. We find that in our parameter regime the error introduced is small as compared to other error sources. The major error is caused by the low contrast and hence the difficulties in edge detection. This error is esti- 


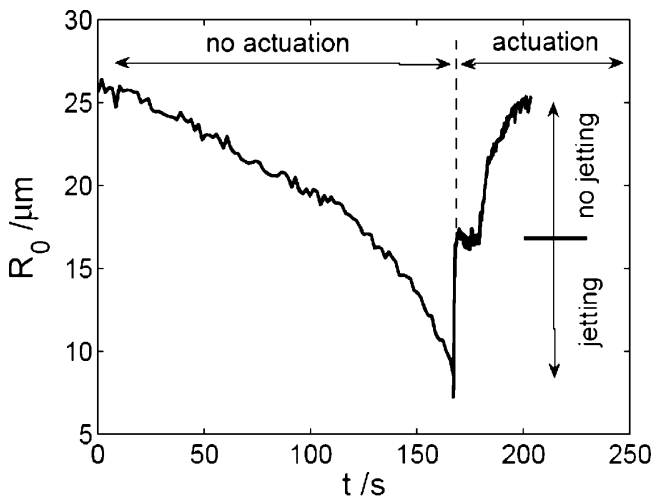

(a)

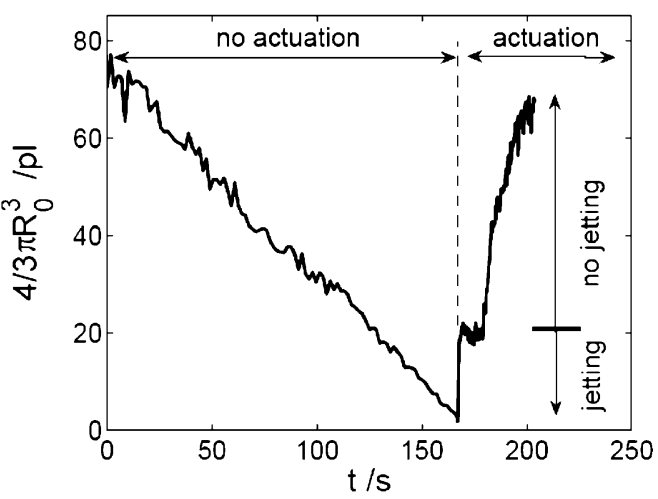

(b)

FIG. 4. Equilibrium radius $R_{0}$ (a) and corresponding bubble volume (b) as a function of time. Up to about $t=165 \mathrm{~s}$ there is no actuation and the bubble dissolves. At $t=165 \mathrm{~s}$ the actuation is started and the bubble, which was nearly fully dissolved, starts to grow by rectified diffusion. From the onset of actuation up to the time that the bubble has reached about $19 \mu \mathrm{m}$ the nozzle is jetting. For larger bubbles the jetting breaks down.

mated to be $\pm 4 \mu \mathrm{m}$ ( 1 pixel). Note that this error is constant for all bubbles, i.e., it is systematic and not statistical. Therefore the shape of the curve, and in particular the existence of the maximum, is not affected by it.

A quantitative explanation of the growth of the bubble as a function of time [Fig. 5(c)] and the dynamics of the bub- ble's position will be presented elsewhere. In particular, we will account for the observed saturation in size as a feedback effect: The grown bubble counteracts the pressure buildup in the channel. Being larger than its resonance size, the bubble is pushed away from the pressure antinode against the nozzle plate.

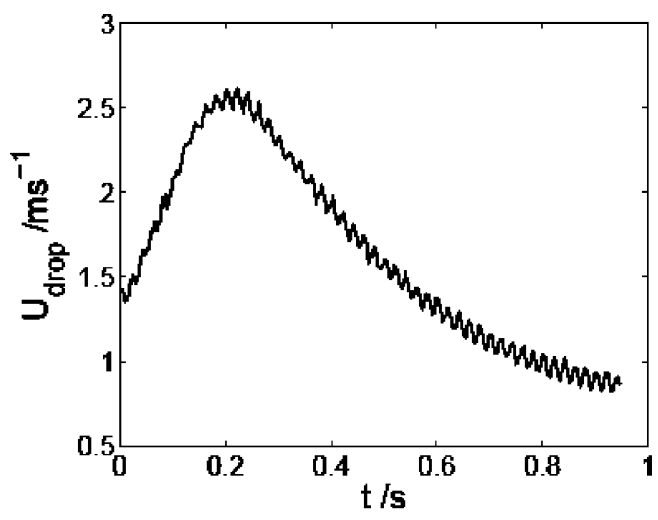

(a)

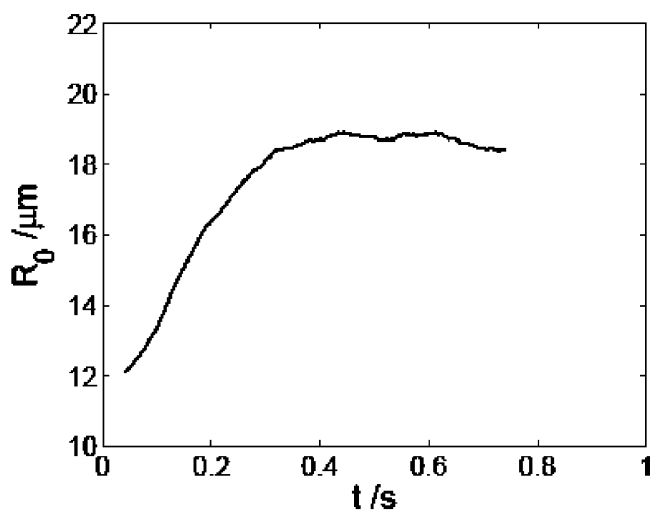

(c)

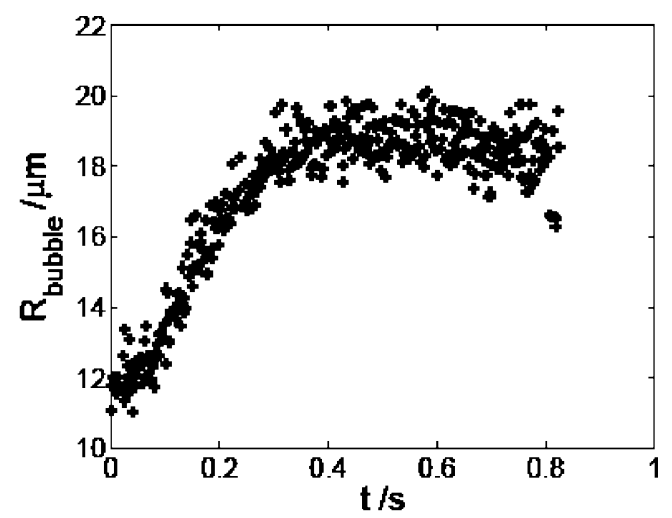

(b)

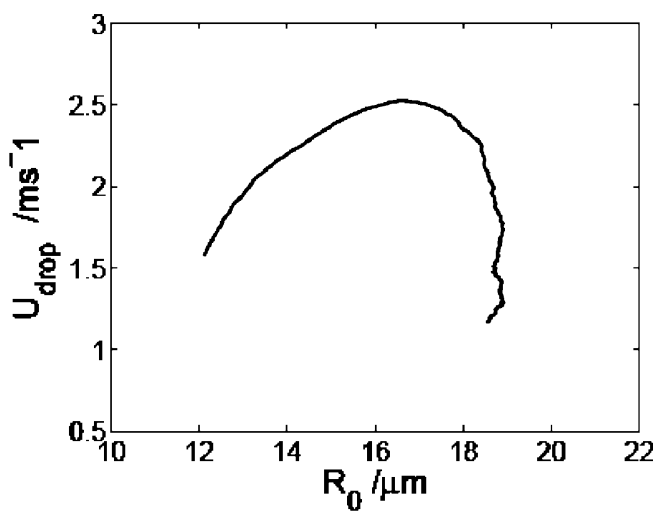

(d)

FIG. 5. (a) The time development of the droplet velocity. At $t=0$ the actuation is started with an air bubble in the ink channel, resulting in an initial droplet velocity enhancement. (b) The radius of the entrapped air bubble over time, showing large variation due to multiple frames during one acoustic cycle. (c) The averaged bubble radius as a function of time. (d) Droplet velocity $u_{\text {drop }}$ as a function of the equilibrium radius $R_{0}$ of the entrapped air bubble. The curve shows a pronounced maximum. 


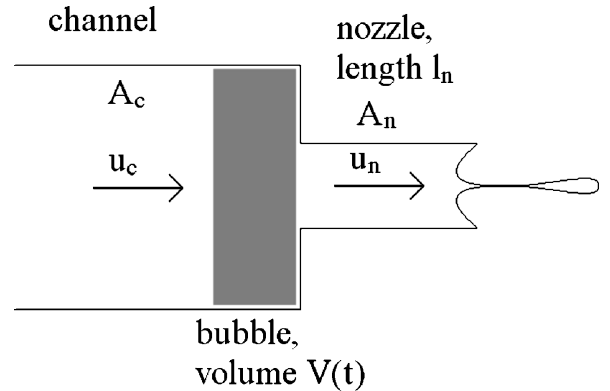

FIG. 6. Sketch of the one-dimensional disk bubble model. Note that in spite of the (compressible) disk bubble we allow for an ink flow between channel and nozzle.

\section{ONE-DIMENSIONAL DISK BUBBLE MODEL}

What is the origin of the maximum in $u_{\mathrm{drop}}\left(R_{0}\right)$ ? It might be expected that the main effect of the bubble in the inkjet nozzle would be to counteract the pressure buildup in the nozzle, as the bubble gets compressed, thus making jetting more difficult. The experimental results, however, show that the effect of the bubble is more subtle, as for small entrained bubbles the droplet velocity increases. To get further insight into the problem, we develop a simple one-dimensional model. It will turn out that the droplet velocity increase is due to a resonance phenomenon.

The nozzle flow and the bubble dynamics are both driven by the channel acoustics. Note the feedback mechanism: The sound-driven bubble emits a pressure wave that propagates into the channel, modifying the channel acoustics and thus ultimately its own dynamics. A complete analysis of the system should therefore comprise the coupling of the channel acoustics to the bubble and nozzle dynamics. For the sake of simplicity, here we neglect the back-effect of the bubble on the channel acoustics.

As shown already in Fig. 2, the channel transfer function has a strong peak at $f=60 \mathrm{kHz}$. To simplify the analysis, in our one-dimensional model we will consider only this dominant frequency and impose a sinusoidal velocity in the channel. Extensions are easily possible, but not necessary to understand the physics of the peak in $u_{\text {drop }}$.

A sketch of the main ingredients of the model is shown in Fig. 6. The channel has cross section $A_{c}$ and the timedependent velocity therein (averaged over the cross section) is $u_{c}(t)$. The nozzle has an effective cross section $A_{n}$, length $l_{n}$, and the time-dependent (average) nozzle velocity is $u_{n}(t)$. In between the channel and nozzle there is a disk-shaped compressible bubble, reflecting the one-dimensional nature of the model. In spite of the fact that, in this simple model, the bubble would actually block the channel, we allow an ink flow in the direction of the nozzle. The relevant feature of the bubble is its compressibility. This disk model is based on Oğuz and Prosperetti's work ${ }^{18}$ who suggested this simplification for large bubbles in tubes.

The viscous friction in the nozzle is approximated by the friction factor of Poiseuille flow. From the Navier-Stokes equation, i.e., from balancing inertia, viscous friction, and the pressure drop through the nozzle between the bubble of pressure $P_{b}$ and the ambient pressure $P_{0}$, one obtains

$$
\frac{\mathrm{d} u_{n}}{\mathrm{~d} t}=\frac{1}{\rho l_{n}}\left(P_{b}-P_{0}\right)-\frac{8 \pi \nu}{A_{n}} u_{n},
$$

where $\nu$ is the kinematic viscosity and $\rho$ the liquid density, assumed to be constant.

We now must connect the nozzle velocity $u_{n}$ with the channel velocity $u_{c}$. In the absence of a bubble, continuity dictates that $A_{n} u_{n}=A_{c} u_{c}$. With the compressible bubble being present, one obtains

$$
\frac{d V_{b}}{d t}=A_{n} u_{n}-A_{c} u_{c} .
$$

The last ingredient is an assumed polytropic relation between the bubble volume $V_{b}(t)$ and its pressure $P_{b}(t)$ :

$$
P_{b}(t)=P_{0}\left(\frac{V_{0}}{V_{b}(t)}\right)^{\gamma} \text {. }
$$

Within the one-dimensional disk bubble model the bubble volume $V_{b}$ can be transformed into an effective thickness $l_{b}(t)=V_{b}(t) / A_{c}$ and the equilibrium volume $V_{0}$ to an equilibrium thickness $l_{0}=V_{0} / A_{c}$.

Upon combining the last two equations we get

$$
\frac{\mathrm{d} P_{b}}{\mathrm{~d} t}=-P_{0} \gamma l_{0}^{\gamma} l_{b}^{(-\gamma-1)}\left(\frac{A_{n}}{A_{c}} u_{n}-u_{c}\right)
$$

or, after linearization around the equilibrium volume [leading to $l_{b}=l_{0}$ in Eq. (4)],

$$
\frac{\mathrm{d} P_{b}}{\mathrm{~d} t}=-\frac{P_{0} \gamma}{l_{0}}\left(\frac{A_{n}}{A_{c}} u_{n}-u_{c}\right) \text {. }
$$

Equations (5) and (1) form a set of two linear first order differential equations for the bubble pressure $P_{b}(t)$ and the nozzle velocity $u_{n}(t)$. Upon eliminating $P_{b}$ we obtain a forced harmonic oscillator equation for the nozzle velocity $u_{n}(t)$ :

$$
\ddot{u}_{n}+2 \beta \dot{u}_{n}+\omega_{0}^{2}\left(R_{0}\right) u_{n}=\omega_{0}^{2}\left(R_{0}\right) \frac{A_{c}}{A_{n}} u_{c} .
$$

The forcing is due to the channel velocity $u_{c}(t)$. The bubblesize dependent eigenfrequency $\omega_{0}$ is given by

$$
\omega_{0}^{2}\left(R_{0}\right)=\frac{\gamma P_{0}}{\rho l_{n} l_{0}} \frac{A_{n}}{A_{c}}=\frac{3 \gamma P_{0} A_{n}}{4 \pi \rho l_{n} R_{0}^{3}}
$$

and the damping coefficient is

$$
\beta=\frac{4 \pi \nu}{A_{n}} .
$$

Both the normalized amplitude $\max _{t}\left(u_{n}\left(t, R_{0}\right)\right) /$ $\max _{t}\left(u_{n}\left(t, R_{0}=0\right)\right)$ [see Fig. 7(a)] and the corresponding phase shift of roughly $\pi$ [Fig. 7(b)] clearly show the resonance behavior. Here we have used $A_{n}=1.3 \times 10^{-9} \mathrm{~m}^{2}$ for the nozzle cross section, $\nu=1.0 \times 10^{-5} \mathrm{~m}^{2} \mathrm{~s}^{-1}$ for the kinematic viscosity, $l_{n}=70 \mu \mathrm{m}$ for the nozzle length, $\rho=1090 \mathrm{~kg} / \mathrm{m}^{3}$ for the ink density, $f_{0}=\omega /(2 \pi)=60 \mathrm{kHz}$ for the dominant frequency, and $P_{0}=101.3 \mathrm{kPa}$ for the ambient pressure. Though the Peclet number $\mathrm{Pe}=R_{0}^{2} f / \kappa$ can approach 1 , for $R_{0}=10 \mu \mathrm{m}, f=60 \mathrm{kHz}$, and the typical heat diffusivity of air 


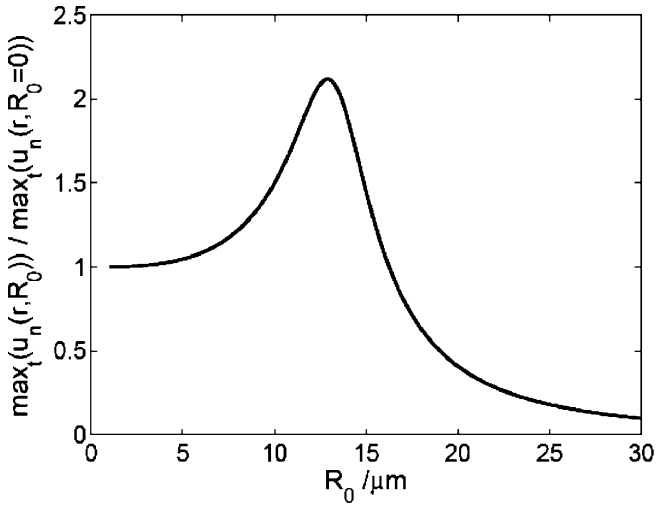

(a)

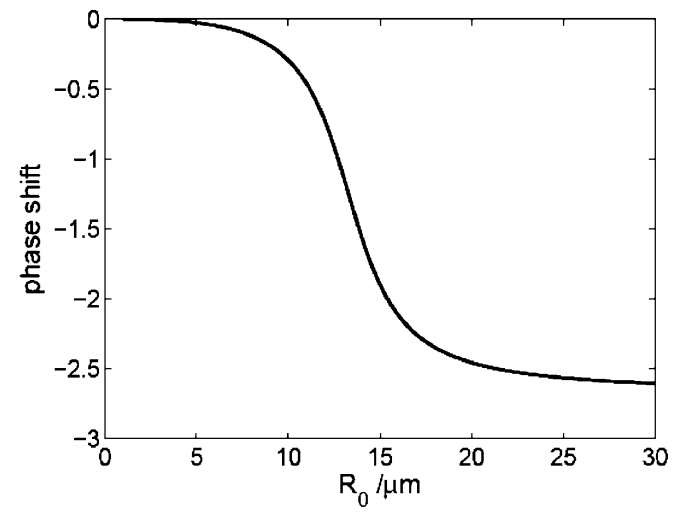

(b)

FIG. 7. (a) Ratio of the maximal nozzle velocity $\max _{t}\left(u_{n}\left(t, R_{0}\right)\right)$ for the case with an entrained bubble to the maximal nozzle velocity $\max _{t}\left(u_{n}\left(t, R_{0}=0\right)\right)$ versus the equilibrium bubble radius $R_{0}$ within the one-dimensional disk bubble model. In (b) the respective phase shift is displayed, clearly signaling the resonance behavior.

$\kappa=15 \times 10^{-6} \mathrm{~m}^{2} / \mathrm{s}$ one obtains $\mathrm{Pe}=0.4$, for simplicity the bubble has been assumed to behave isothermally throughout the cycle, $\gamma=1$. Taking the adiabatic value $\gamma=7 / 5$ only leads to a small shift of the maximum.

The location of the nozzle velocity maximum in Fig. 7(a) can straightforwardly be calculated, leading to the resonance radius

$$
R_{0}^{\mathrm{res}}=\left(\frac{3 \gamma A_{n}^{3} P_{0}}{4 \pi \rho l_{n}\left(\omega^{2} A_{n}^{2}+64 \nu^{2} \pi^{2}\right)}\right)^{1 / 3} .
$$

Using the above values for the parameters, we obtain $R_{0}^{\text {res }} \approx 13 \mu \mathrm{m}$, in agreement with Fig. 7(a). The phase shift at resonance is not exactly $\pi$ as a result of viscous friction in the nozzle.

\section{COMPARISON}

To compare the results of the model with the data, the resulting droplet velocity must be calculated from the nozzle velocity. This can be done with the method described by Dijksman. ${ }^{3}$

The droplet formation is assumed to start when the nozzle velocity becomes positive, and to end when the kinetic energy density of the forming droplet becomes larger than the average kinetic energy density of the fluid flowing out of the nozzle. The ink that is outside the nozzle at that time is assumed to form the droplet. The droplet leaves the nozzle with a kinetic energy equal to the kinetic energy of the forming droplet at that time. The corrections for viscous and capillary effects during the formation of the tail are neglected. A parabolic flow profile is assumed.

For a sinusoidal driving velocity at a frequency of $f=60 \mathrm{kHz}$ and the geometry of the experimental setup, the droplet velocity monotonously depends on the nozzle velocity amplitude, see Fig. 8. Therefore, a single peak in the nozzle velocity causes a single peak in the droplet velocity. Physically, the threshold observed in Fig. 8 of course reflects the finite amount of energy being necessary to form the surface of a drop.
Using this droplet formation model, the droplet velocity can be calculated as a function of the bubble radius, allowing for a direct comparison of the model results with the experimental ones, see Fig. 9. The amplitude of the channel flow was chosen to yield the experimentally determined droplet velocity in the absence of a bubble.

The bubble radius causing a maximum of $2.5 \mathrm{~m} / \mathrm{s}$ in the droplet velocity was experimentally found to be $R_{0}=17 \pm 4 \mu \mathrm{m}$. In contrast, the disk bubble model gives a maximum in the droplet velocity at $R_{0}=13 \mu \mathrm{m}$ (for a mean nozzle radius of $20 \mu \mathrm{m}$ ), quantitatively slightly off, but in reasonable agreement, considering the experimental systematic error of $4 \mu \mathrm{m}$ in the estimate of the bubble radius and the simplifications of the model. The estimated maximum velocity is $5.3 \mathrm{~m} / \mathrm{s}$, about twice the measured value.

For a nozzle radius of $15 \mu \mathrm{m}$, the calculated maximum in the droplet velocity shifts to $R_{0}=9 \mu \mathrm{m}$, which is outside the uncertainty interval, see the dotted line in Fig. 9. However, the maximum droplet velocity of $3.0 \mathrm{~m} / \mathrm{s}$ is closer to the measured velocity maximum.

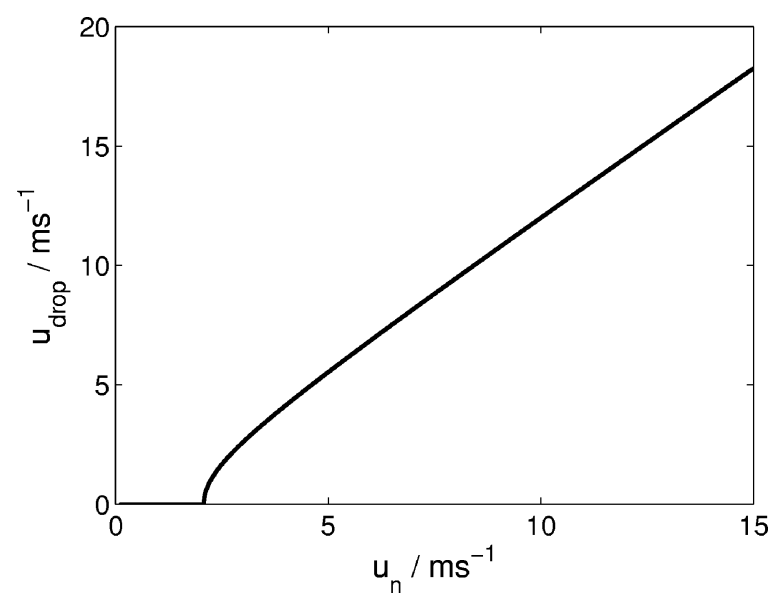

FIG. 8. The droplet velocity calculated from the nozzle velocity according to Dijksman (Ref. 3) assuming a parabolic velocity profile. A nozzle velocity amplitude of at least $u_{n}=2.0 \mathrm{~m} / \mathrm{s}$ is required to form a droplet. 


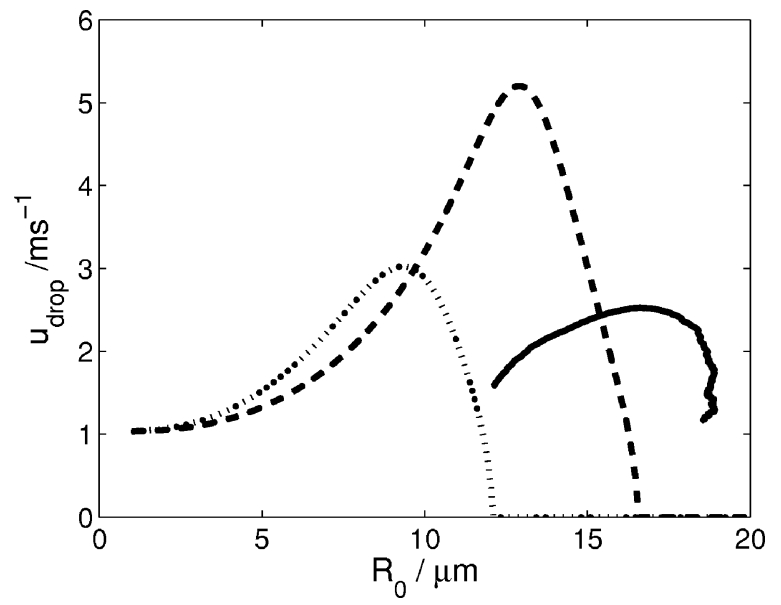

FIG. 9. The droplet velocity $u_{\text {drop }}$ as a function of the equilibrium radius $R_{0}$. The experimental results (solid) show a maximum in droplet velocity at $R_{0}=17 \mu \mathrm{m}$. The numerical model displays a maximum at $R_{0}=13 \mu \mathrm{m}$ (dashed) when the average nozzle radius $20 \mu \mathrm{m}\left(A_{n}=1.3 \cdot 10^{-9} \mathrm{~m}^{2}\right)$ is taken as the relevant transversal length scale of the nozzle, and a maximum at $R_{0}=9 \mu \mathrm{m}$ (dotted) when the minimal nozzle radius of $15 \mu \mathrm{m}$ $\left(A_{n}=7.1 \cdot 10^{-10} \mathrm{~m}^{2}\right)$ is taken instead.

The results of the model are sensitive to the exact value taken for the nozzle cross section. However, the shapes of the predicted and measured peaks are very similar. Even the sharp drop in droplet velocity for bubble sizes slightly below the size where droplet formation stops is represented in the one-dimensional disk model where it is caused by the infinite slope of the droplet velocity as a function of nozzle velocity.

Very small bubbles $\left(R_{0}<1 \mu \mathrm{m}\right)$ do not influence the droplet velocity. However, such bubbles are too small to be optically detected.

\section{CONCLUSIONS AND OUTLOOK}

The influence of the bubble size on the droplet velocity in an inkjet printhead was measured and modeled with a simple one-dimensional disk bubble model. As expected, small bubbles have no effect on the droplet velocity. The main finding of our work is that for intermediate bubble radii the droplet velocity is increased by the bubble. This remarkable phenomenon is caused by a resonance in the volume oscillations of the bubble, which are driven by the flow in the nozzle. Large bubbles cause nozzle failure. The droplet formation ceases abruptly as the bubble radius increases.

After these mechanisms have been understood, our research on the problem will go in several directions. First of all, we will extend the one-dimensional disk bubble model to a full, three-dimensional and two-way coupled numerical model. We hope that with such a model the effect of the bubble size and of its exact position on the droplet velocity can be quantitatively captured. Next, we would like to quantitatively understand the bubble growth by rectified diffusion and its dynamics in the ink channel. While rectified diffusion is quantitatively understood in still liquid in the bulk, both the liquid flow along the bubble and the constrained geometry in the nozzle strongly affect the bubble's growth and must be accounted for.

While we have revealed that small particles and an ink layer on the nozzle can lead to air entrainment, ${ }^{12}$ it is not yet clear why at certain frequencies and for certain shapes the nozzle is more vulnerable to air entrainment than at others. A resonance mechanism between the driving frequency and the eigenfrequency of the meniscus may be the origin for this observed strong dependence, and we plan to examine this conjecture.

From an application point of view the ultimate goal must of course be to avoid air entrainment or to immediately flush out the bubble once it has been entrained.

\section{ACKNOWLEDGMENTS}

We thank the staff of Océ Technologies B.V. for support, in particular Jack Bisschop for construction of the modified printhead. This work is part of the research program of the Stichting voor Fundamenteel Onderzoek der Materie (FOM), which is financially supported by the Nederlandse Organisatie voor Wetenschappelijk Onderzoek (NWO) and by Océ Technologies B.V.

${ }^{1}$ C. Williams, "Ink-jet printers go beyond paper," Phys. World 19, 24 (2006).

${ }^{2}$ H. P. Le, "Progress and trends in ink-jet printing technology," J. Imaging Sci. Technol. 42, 49 (1998).

${ }^{3}$ J. F. Dijksman, "Hydrodynamics of small tubular pumps," J. Fluid Mech. 139, 173 (1984).

${ }^{4}$ D. Y. Shin, P. Grassia, and B. Derby, "Oscillatory limited compressible fluid flow induced by the radial motion of a thick-walled piezo-electric tube," J. Acoust. Soc. Am. 114, 1314 (2003).

${ }^{5}$ J. M. Meacham, M. J. Varady, F. L. Degertekin, and A. G. Fedorov, "Droplet formation and ejection from a micromachined ultrasonic droplet generator: Visualization and scaling," Phys. Fluids 17, 100605 (2005).

${ }^{6} \mathrm{~W}$. Berggren, M. Westphall, and L. Smith, "Single-pulse nanoelectrospray ionization," Anal. Chem. 74, 3443 (2002).

${ }^{7}$ A. Chen and O. Basaran, "A new method for significantly reducing drop radius without reducing nozzle radius in drop-on-demand drop production," Phys. Fluids 14, L1 (2002).

${ }^{8}$ S. Elrod, B. Hadimioglu, B. Khuri-Yakub, E. Rawson, E. Richley, C. Quate, N. Mansour, and T. Lundgren, "Nozzleless droplet formation with focused acoustic beams," J. Appl. Phys. 65, 3441 (1989).

${ }^{9}$ J. F. Dijksman, "Hydro-acoustics of piezo-electrically driven ink-jet print heads," Flow, Turbul. Combust. 61, 211 (1999).

${ }^{10}$ J. D. Brock, I. M. Cohen, I. P. Ivanov, H. P. Le, and J. Roy, "Oscillations of an air bubble in an ink jet," J. Imaging Technol. 10, 127 (1984).

${ }^{11}$ N. P. Hine, "Deaeration system for a high-performance drop-on-demand ink jet," J. Imaging Technol. 17, 223 (1991).

${ }^{12}$ J. de Jong, H. Reinten, M. van den Berg, H. Wijshoff, M. Versluis, G. de Bruin, and D. Lohse, "Air entrapment in piezo-driven inkjet printheads," J. Acoust. Soc. Am. 120, 1257 (2006).

${ }^{13}$ C. E. Brennen, Cavitation and Bubble Dynamics (Oxford University Press, Oxford, 1995).

${ }^{14}$ M. M. Fyrillas and A. J. Szeri, "Dissolution or growth of soluble spherical oscillating bubbles," J. Fluid Mech. 277, 381 (1994).

${ }^{15}$ S. Hilgenfeldt, D. Lohse, and M. P. Brenner, "Phase diagrams for sonoluminescing bubbles," Phys. Fluids 8, 2808 (1996).

${ }^{16}$ T. G. Leighton, The Acoustic Bubble (Academic, London, 1994).

${ }^{17} \mathrm{H}$. Tijdeman, "On the propagation of sound waves in cylindrical tubes," J. Sound Vib. 39, 1 (1975).

${ }^{18} \mathrm{H}$. N. Oğuz and A. Prosperetti, "The natural frequency of oscillation of gas bubbles in tubes," J. Acoust. Soc. Am. 103, 3301 (1998).

${ }^{19}$ Micronit Microfluidics B.V., P.O. Box 545, 7500 AM Enschede, The Netherlands. 